\title{
L'Ouganda en campagne précoce pour 2011
}

\section{Florence Brisset-Foucault}

\section{(2) OpenEdition}

\section{Journals}

Édition électronique

URL : https://journals.openedition.org/eastafrica/570

DOI : 10.4000/eastafrica. 570

ISSN : 2790-1076

\section{Éditeur}

IFRA - Institut Français de Recherche en Afrique

\section{Édition imprimée}

Date de publication : 1 janvier 2010

Pagination : 5-41

ISSN : 2071-7245

\section{Référence électronique}

Florence Brisset-Foucault, « L'Ouganda en campagne précoce pour 2011 », Les Cahiers d'Afrique de l'Est / The East African Review [En ligne], 42 | 2010, mis en ligne le 07 mai 2019, consulté le 09 décembre 2021. URL : http://journals.openedition.org/eastafrica/570 ; DOI : https://doi.org/10.4000/eastafrica. 570

Ce document a été généré automatiquement le 9 décembre 2021

Les Cahiers d'Afrique de l'Est / The East African Review 


\title{
L'Ouganda en campagne précoce pour 2011
}

\author{
Florence Brisset-Foucault
}

1 2008-2009 fut un bon cru en termes de "sagas", pour reprendre les mots des journalistes ougandais. L'année s'est déroulée au rythme d'affaires de corruption, de mœurs et de trahison politique qui se croisent et s'alimentent. Affaires sur lesquelles journalistes, opposants de tous bords et membres du gouvernement ou du parti au pouvoir rebondissent, à la poursuite de leur propre agenda.

2 Le lancement précoce de la campagne électorale, en vue des élections présidentielles et parlementaires de 2011, ne fut pas étranger à cette inflation de scandales. En 2008, dans les meetings politiques du NRM (National Resistance Movement), le parti au pouvoir, les arguments défendant le bien-fondé d'un quatrième mandat du président Yoweri Kaguta Museveni étaient sur toutes les lèvres. Les militants avaient déjà quatre doigts de la main droite bien tendus, symbolisant leur soutien pour une nouvelle candidature de leur champion. Sur le terrain, des groupes de mobilisation pour la promotion du quatrième mandat ont été mis en place ${ }^{1}$. Les différents partis se concertent sur le mode de désignation interne des candidats ${ }^{2}$. Les pronostics vont bon train sur le retour de politiciens exilés prêts à revenir en Ouganda pour affronter Museveni dans les urnes ${ }^{3}$.

3 Le signal de départ de la campagne a été donné avec la grande tournée de Museveni dans le pays en 2008. Ces déplacements visaient officiellement à promouvoir l'initiative «Prospérité pour tous » (Bonna Bagaggawale), le programme économique défendu par le NRM lors de la campagne électorale de 2006, qui vise à réduire la pauvreté en développant notamment la marchandisation de la production agricole domestique. Le président a porté une attention particulière à l'Est du pays et au Bunyoro (un royaume de l'Ouest du pays), qui font partie des régions clés dans l'affrontement à venir.

4 En février 2009, dans un discours prononcé à Arua, l'une des principales villes de la région du West Nile (Nord-Ouest du pays et bastion de l'opposition), le président a souligné les répercussions économiques et politiques du choix des électeurs : "Un vote pour Besigye est un vote pour la guerre civile. Ce n'est pas une plaisanterie, c'est une chose sérieuse: si vous votez pour le NRM votre zone sera développée $e^{4}$." 
5 Le Forum for Democratic Change (FDC), principal parti d'opposition, n'est pas en reste. Kiiza Besigye, adversaire de Museveni en 2001 et 2006, a parcouru tout le pays lors de l'été 2008. En février 2009, le parti a désigné ses nouveaux secrétaires nationaux et réélu Kiiza Besigye président.

6 La perspective d'une compétition électorale difficile détermine les stratégies politiques du cœur du pouvoir, sur les plans interne et externe. En 2011, cela fera 25 ans que Yoweri Kaguta Museveni est au pouvoir. Un anniversaire malvenu aux yeux de ceux qui, en Ouganda comme ailleurs (notamment parmi les bailleurs de fonds), se soucient de l'état de la démocratie dans ce petit pays d'Afrique orientale. Face à cette perte de légitimité, le régime doit repenser ses appuis et chercher de nouvelles sources de popularité. Déjà, avec l'organisation, en novembre 2007, du Commonwealth Head of Government Meeting (CHOGM), le régime a cherché à redorer son blason à l'extérieur comme à l'intérieur, la conférence étant l'occasion de lancer un programme ambitieux d'investissements publics.

7 La longueur du règne de Museveni excite également les convoitises de ses cadets, et notamment des membres les plus influents du NRM qui espèrent bien pouvoir accéder au sommet de l'État dans un futur proche.

Les tensions se cristallisent sur plusieurs dossiers. Le premier est constitué de conflits internes au NRM, sur fond d'affaires de corruption. Le second touche l'un des piliers du système politique : la remise en cause croissante de l'alliance entre le gouvernement central et le royaume du Buganda, sur laquelle avait reposé la victoire militaire de la NRA en 1986 et, en grande partie, la consolidation du régime NRM depuis lors. Le troisième dossier est bien sûr le conflit qui oppose le gouvernement aux rebelles de l'Armée de résistance du Seigneur (Lord's Resistance Army, LRA) depuis le milieu des années 1980. Enfin, la question des libertés publiques se pose de manière de plus en plus aiguë. Malgré le passage au multipartisme en juillet 2005, les menaces sur les opposants et les médias persistent. Ces acteurs, non exempts de conflits internes, parviennent cependant, avec plus ou moins de succès, à faire entendre une voix critique au sein de l'espace public ougandais. Après un passage en revue de ces quatre dossiers, nous livrerons à la fin de cette contribution quelques éléments sur les ressorts de la mobilisation pour la défense des droits des homosexuels. Ce mouvement social relativement inédit en Afrique a marqué l'actualité ougandaise depuis plusieurs années et plus particulièrement depuis 2007.

\section{Les conflits internes au National Resistance Movement}

9 De nombreux acteurs, de la LRA aux royalistes ganda en passant par les partis d'opposition, cherchent à remettre en cause la mainmise du NRM sur le pays. Cependant, l'une des principales caractéristiques du régime depuis 1986 reste l'importance des menaces endogènes et de la critique interne au parti ${ }^{5}$. Bien sûr, cette séparation entre le "dedans » et le "dehors " n'est que schématique, puisque l'un et l'autre s'alimentent et ne peuvent être compris séparément. Cependant, pour la clarté de l'exposé, on s'attardera dans cette première section sur les difficultés propres au parti dominant et sur les ressorts des conflits entre les élites au pouvoir. 


\section{L'influence des scandales de corruption sur l'architecture du NRM}

10 L'affaire du détournement de fonds de l'Action globale pour la vaccination et l'immunisation (GAVI), a continué à faire des remous pendant toute l'année 2008. Le Major Général Jim Muhwezi, ancien ministre de la Santé, membre du Comité exécutif central du NRM, secrétaire de la ligue des vétérans du parti, ainsi que d'autres importantes figures du NRM (Mike Mukula, Alex Kamugisha, Alice Kaboyo), furent arrêtés en mai 2007 suite à une enquête de l'Inspecteur général du gouvernement (IGG). Ils sont accusés d'avoir détourné 700 millions de shillings (280 000 euros). Mike Mukula (vice-chairman du NRM pour l'Ouganda de l'Est) et Jim Muhwezi ont déjà été mis en cause en 2006 dans une autre affaire, celle des détournements des Fonds globaux contre le sida, le paludisme et la tuberculose. La procédure judiciaire pour cette affaire est en cours et prise en charge par la nouvellement créée Cour Anti-Corruption. Écartés du pouvoir suite au scandale, Muhwezi et les siens ont alimenté une fronde interne au NRM. Ils affirment que cette affaire a été montée par leurs adversaires au sein du parti afin de les éliminer de la compétition. L'affaire est d'autant plus complexe que la première dame,

11 Janet Museveni, est accusée par les personnes mises en cause d'avoir également bénéficié d'une part des fonds GAVI. La procédure à l'encontre de Muhwezi et de ses comparses a été interrompue lorsque ces derniers ont déposé une plainte auprès de la Cour constitutionnelle, avançant que l'IGG avait mené l'enquête à la demande du président, alors que cette institution est censée être indépendante.

12 La fronde interne au NRM menée par Muhwezi a pris une ampleur importante suite à la révélation d'une autre affaire impliquant les fonds de la Sécurité sociale nationale (NSSF), i.e. les cotisations des travailleurs pour leur retraite. La « saga » NSSF est sortie dans la presse en août 2008. Également appelée l'affaire Temangalo, elle implique le ministre de la Sécurité et secrétaire général du NRM, Amama Mbabazi, souvent décrit comme le dauphin de Museveni et adversaire de Muhwezi au sein du NRM. Le contentieux réside dans le fait que le NSSF a acheté un terrain, dont une partie appartient à Mbabazi, situé à Temangalo (dans le district de Wakiso à l'Ouest de Kampala) de 400 acres pour la somme de 11 milliards de shillings (4 400000 euros). Plusieurs questions ont alors été posées autour desquelles le débat a fait rage pendant plusieurs mois. Y a-t-il eu violation des lois d'attribution des marchés publics ? Le prix payé pour le terrain était-il plus haut que celui du marché ? Le NSSF a-t-il acheté ce terrain sous pression politique?

13 Mbabazi et le ministre des Finances, Ezra Suruma (qui a la tutelle du NSSF) se sont retrouvés sous le feu des critiques de la presse, de l'opposition et de certains des députés NRM. Muhwezi était l'un des principaux inquisiteurs. Une partie des membres du caucus NRM au sein du Parlement ont demandé la démission de Mbabazi. L'ampleur du scandale a poussé les parlementaires à organiser une enquête dont a été chargé le Comité parlementaire sur les commissions de droit public et les entreprises publiques. Le comité s'est profondément divisé. Six parlementaires n'étaient pas d'accord avec les conclusions d'un premier rapport, qui pointait du doigt des violations des lois d'attribution des marchés publics et le fait que le prix d'achat du terrain était plus haut que les prix du marché. Ces six parlementaires ont donc décidé d'écrire un «minority report » qui se désolidarisa de ces conclusions. Mbabazi a été sommé de défendre sa position devant le caucus du NRM au Parlement, devant le comité parlementaire qui 
faisait l'enquête et devant les autorités du parti. Auditions lors desquelles il a nié les accusations dont il faisait l'objet. Les comités parlementaires n'ayant pas de pouvoir disciplinaire, les rapports ont été transmis à l'IGG en mars 2009. Le parti au pouvoir s'est donc profondément divisé dans cette affaire entre les pro et les anti-Mbabazi, menés par Muwhezi mais aussi par un autre poids lourd du mouvement et de la guerre du bush (1981-1986), le général Kahinda Otafiire.

La justice et l'IGG doivent toujours statuer sur ces différentes affaires. Que les différentes accusations soient fondées ou pas, ces tirs croisés sur le registre de la moralité politique et de la corruption habillent et alimentent des rivalités politiciennes entre plusieurs factions. L'une des sources de mécontentement réside dans les degrés divers de sévérité du président selon les affaires, certains ministres se voyant écartés du gouvernement après avoir été soupçonnés de corruption (Muhwezi) et d'autres non (Mbabazi).

La presse joue toujours un rôle de premier plan dans le dévoilement de ces affaires, qui mettent en jeu des sommes publiques importantes. Cependant, l'économie des "scoops» et des révélations de la part de "sources fiables et anonymes» est stratégiquement gérée par ces mêmes cadres politiques. Dans ce chassé-croisé, les journalistes gagnent en tant que corps professionnel à réaffirmer leur rôle de chien de garde, mais ils cherchent également à ne pas se laisser manipuler. La plupart ne sont pas dupes des intérêts des sources à faire sortir telle ou telle affaire et cherchent à développer une réflexivité plus grande sur l'opportunité de publier ou non ces révélations ${ }^{6}$.

Les tensions entre les différentes factions du NRM n'ont fait que s'accentuer avec le remaniement ministériel de février 2009 et l'approche de la rencontre des membres du Conseil exécutif national (NEC) du parti en mai 2009. Les protagonistes s'affrontant à grands coups de conférences de presse et de SMS anonymes là encore largement transmis aux journalistes ${ }^{7} \ldots$

\section{Les rebondissements de la critique interne}

17 Les différentes affaires qui ont marqué l'année 2008, et plus particulièrement le scandale NSSF, ont donc vu la contestation interne au NRM se renforcer. Certains députés, les "députés rebelles » ou "rebelles du NRM », affichent au grand jour des positions hostiles au président et à Amama Mbabazi, que ce soit dans la presse ou au sein du Parlement. Ils sont appuyés plus ou moins ouvertement par certains «historiques », des membres fondateurs du NRM, souvent vétérans de la guerre du bush$^{8}$. Les «députés rebelles» ne sont cependant pas uniquement composés de vétérans et comptent également dans leurs rangs des «jeunes turcs» (Banyenzaki, Baryomunsi) qui demandent une alternance générationnelle au sein du parti.

Ce type de ruptures est loin d'être un phénomène nouveau. De nombreux "historiques» ont quitté le NRM et rejoint les critiques de Museveni depuis une dizaine d'années. Les dissensions et le débat interne ont toujours été forts au sein du Mouvement, qui, avant 2005, n'était pas censé être un parti mais englober tous les citoyens ougandais. Le NRM a connu plusieurs fractures depuis sa création, notamment au moment du processus constitutionnel au milieu des années 1990, en 2001 lors de la candidature Besigye et en 2005 , lors de la modification de la Constitution qui devait permettre à Museveni de briguer un troisième mandat. Les opposants les plus 
importants dans l'Ouganda contemporain sont issus du cœur du NRM (Kiiza Besigye, Mungisha Muntu, Jaberi Bibandi-Ssali). Dans les $6^{\mathrm{e}}$ et $7^{\mathrm{e}}$ parlements (1996-2001; 2001-2006), il existait également des groupes de pression formés par des «députés rebelles ": l'Association des jeunes parlementaires et le Forum pour le plaidoyer parlementaire (Parliamentary Advocacy Forum qui a rejoint en 2004 l'Agenda de réforme de Besigye pour former le FDC) ${ }^{9}$. Le contexte multipartiste pose cependant de manière encore plus aiguë la question de la discipline politique au sein d'une même formation partisane.

La négociation des alliances entre les élites au pouvoir ne concerne pas uniquement le personnel politique. Le mois de juillet 2008 a été marqué par les déclarations du Brigadier Henry Tumukunde, vétéran de la guerre du bush et ancien chef de l'Organisation intérieure de sécurité (ISO). En juin 2003, le brigadier Tumukunde avait quitté l'ISO sans être redéployé. Il a ensuite été forcé de démissionner de son poste de parlementaire en mai 2005 après avoir critiqué l'armée et le gouvernement sur deux radios de Kampala. Il a passé un an en détention, accusé de répandre des discours de haine et d'avoir rompu le code militaire. Lors d'un enterrement, en juillet 2008, il a, en compagnie de Muhwezi, une fois de plus critiqué le gouvernement et l'armée, dénonçant des persécutions dont ils seraient l'objet. Ces déclarations conjointes sont venues alimenter l'idée d'un détachement politique des vétérans contre Museveni, dans l'objectif des élections de 2011, comme cela a déjà été vu en 2001 avec la candidature Besigye.

20 Les déclarations de Tumukunde ont la particularité d'avoir été en partie émises sur le registre ethnique, Tumukunde ayant déclaré, lors de ce fameux enterrement: « Touchez à un Bahororo et vous nous avez tous attaqués. " Les Bahororo, communauté reconnue par la Constitution de 1995, vivent dans l'extrême Sud-Ouest du pays, dans la région de Kigezi et le district de Rukungiri (où se trouve la circonscription de Muhwezi), tout proche de la région d'origine de Museveni (lui-même un Munyankole). Besigye, Muhwezi et Tumukunde, qui viennent tous les trois du district de Rukungiri, sont censés faire partie de ce groupe, ce qui, dans le discours de Tumukunde, vient appuyer l'idée d'une persécution ${ }^{10}$.

21 Par ailleurs, le 27 mars 2008, le Major Général Kazini, a été condamné à trois ans de prison dans le cadre de l'affaire des "soldats fantômes" pour avoir détourné 61 millions de shillings sous forme de salaires fictifs. ${ }^{11}$. Kazini est un ancien Commandant de l'armée (2001-2003), commandant en 1998 de l'opération « Mountain Rescue » contre les rebelles de l'ADF, déployé ensuite en RDC, où il est soupçonné de s'être livré à des activités de pillage.

Il est intéressant de noter l'importance persistante des allusions à la guerre du bush dans les oppositions politiques. La question «Qui s'est battu? » revient régulièrement dans le débat public, avec de nombreux feuilletons relatant les années de guérilla dans les journaux ou la publication de listes des $"$ héros de guerre $»^{12}$. L'opposition entre Kahinda Otafiire et Muhwezi, connus pour leur implication dans la guerre d'une part et Amama Mbabazi, qui faisait partie de la délégation étrangère du NRM à ce moment-là d'autre part, n'est pas neutre en ce sens ${ }^{13}$. 


\section{Le remaniement ministériel de février 2009}

23 Le 16 février 2009, le président a opéré un important remaniement ministériel. Cette opération était censée être une réaction à l'affaire NSSF. Les leçons tirées du scandale n'ont visiblement pas été les mêmes pour Museveni et pour ses opposants. Au lieu de punir les responsables présumés des détournements de fonds, le président a choisi de renforcer le camp d'Amama Mbabazi et d'opérer un resserrement du gouvernement autour de ses fidèles. Le nouveau cabinet est pléthorique. Il compte 70 membres (en comptant les ministres du cabinet et les ministres d'État). Le ministre de la Sécurité en sort largement renforcé. Non seulement il n'est pas écarté, mais il n'est pas rétrogradé, restant à la tête d'un ministère clé malgré la polémique et la demande de démission le concernant portée par plusieurs députés NRM. Ses alliés ont été placés à la tête de plusieurs ministères et ses adversaires éloignés.

Le remaniement a remis de l'huile sur le feu sur le conflit entre Mbabazi et Kahinda Otafiire, jusqu'alors ministre du Gouvernement local. Ce dernier faisait partie des critiques de Mbabazi dans le scandale NSSF. Otafiire s'est vu attribuer le petit ministère du Commerce, du Tourisme et de l'Industrie. Il a été remplacé au Ministère du Gouvernement local par Adolf Mwesige, un allié de Mbabazi.

Kabakumba Matsiko, qui avait soutenu Mbabazi, a été nommée ministre de l'Information et de l'orientation nationale. Hope Mwesige, l'une des grandes alliées de Mbabazi, est devenue ministre de l'Agriculture. Parmi les personnalités impliquées dans le scandale NSSF, seul le ministre des Finances, Ezra Suruma, ministre de tutelle du NSSF, a été mis à la porte.

Salim Saleh, demi-frère du président et jusqu'alors ministre de la Micro-finance, a été nommé conseiller du président pour la Défense. Une manière d'éviter que deux personnes de la proche famille du président soient au gouvernement, puisque l'autre élément marquant de ce remaniement reste la nomination de l'épouse du président, Janet Museveni, au lointain ministère pour la région Karamoja.

\section{Les ressorts de l'affaire Mwondha}

Les mois de février à juin 2009 ont été dominés par l'affaire Faith Mwondha. $\mathrm{M}^{\text {me }}$ Mwondha occupe le poste d'Inspecteur général du gouvernement (IGG) depuis 2005. Ce puissant organe de contrôle destiné à la lutte contre la corruption a été créé dès $1986^{14}$.. L'article 225 de la Constitution décrit les fonctions de l'IGG comme étant de veiller au respect de la loi et de la justice dans l'administration, d'éliminer les actes de corruption et les abus de pouvoir, de veiller au respect du code de conduite des dirigeants (chap. 14 de la Constitution). L'IGG a notamment pour fonction d'enquêter sur toute action d'un fonctionnaire ou représentant de l'État dans le cadre de sa fonction. L'IGG peut lancer une enquête de sa propre initiative ou suite à une plainte déposée par n'importe quel membre du public. Faith Mwondha a été nommée en 2005. Lors de son mandat, elle n'hésita pas à aller à la confrontation et ne mâcha pas ses mots avec ceux qu'elle appelle les « corrompus ». Elle fut notamment directement à l'origine de l'arrestation, en mai 2007, de Muhwezi, Mukula et leurs comparses, ce qui lui a valu d'être accusée de participer aux projets d'élimination des concurrents de Museveni au sein du NRM. De fait, elle n'est pas intervenue dans l'affaire Temangalo et s'est engagée dans un conflit avec Kahinda Otafiire ${ }^{15}$. $\mathrm{M}^{\text {me }}$ Mwondha a eu une influence déterminante 
dans la mise en place de la Cour anti-corruption. Sous son mandat, l'IGG a eu une activité intense, en particulier au niveau local. Aujourd'hui, l'institution s'est vue transférer le dossier Temangalo par les parlementaires. années, elles se sont intensifiées depuis 2007 via la polémique autour d'un projet de réforme foncière. Ces désaccords sont au centre de projets concurrents de mobilisation et pourraient peser fortement dans la balance lors des élections de 2011. 


\section{Un conflit ancien}

33 Le statut des terres dans la région centrale de l'Ouganda est au centre d'affrontements politiques depuis la colonisation. Lors des débats qui ont précédé la restauration des royaumes en 1993, l'un des points de contentieux était la restitution de biens fonciers et immobiliers (les Baganda parlent de "nos choses ", ebyaffe) appartenant au royaume jusqu'à sa suppression par Obote en 1967. À ces questions foncières s'ajoute celle du statut politique du Royaume par rapport à l'État central. Lors des débats préalables à la mise en place de la nouvelle Constitution, dans les années 1990, des délégués ganda de l'Assemblée constituante ont milité pour la mise en place le système dit du Federo, autorisant un certain degré d'autonomie administrative et politique du royaume ${ }^{17}$.

Il faut remonter au début du siècle pour bien comprendre les ressorts de la polémique. En 1900, les Britanniques signèrent un accord avec le royaume du Buganda ${ }^{18}$. Cet accord a créé plusieurs statuts fonciers. Parmi ces terres, 8000 miles $^{2}$ ont été attribués à des chefs ganda. 9000 miles $^{2}$ ont été attribués à la Couronne ${ }^{19}$. Ces 9000 miles $^{2}$ ont été réduits au fur et à mesure de l'histoire, entre autres du fait de la perte de terres au profit du Bunyoro en 1964 (les "comtés perdus ») ${ }^{20}$. Les 8000 miles $^{2}$ ne sont pas toujours restés dans les familles des premiers notables ganda qui se les étaient vus attribuer, certaines terres ayant été vendues à des Baganda ou des non-Baganda.

En 1967, les royaumes sont abolis par Obote. Les terres de la couronne (les fameux 9000 miles $^{2}$ moins les " comtés perdus » réattribués au Bunyoro en 1964) ont été mises sous l'autorité de l'État central. Aujourd'hui, dans la foulée de la restauration de 1993, certains militants royalistes ganda voudraient voir la restitution de ces 9000 miles $^{2}$ à la couronne. Ces terres sont encore sous le contrôle de l'État et les royalistes arguent que le projet actuel d'amendement de la loi sur les terres de 1998 rend cette perspective de restitution encore plus lointaine. Par ailleurs, une partie de ces fameux 9000 miles $^{2}$ reste sur le territoire du Bunyoro, ce qui vient mettre de l'huile sur le feu. Le fait que le gouvernement détient encore des biens appartenant au royaume accroît les tensions.

La polémique actuelle concerne également les terres n'appartenant pas à la Couronne

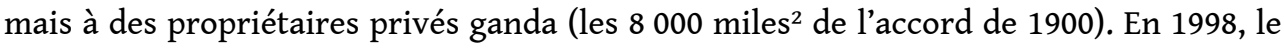
gouvernement fit passer une première loi sur les terres. Cette loi cherchait à renforcer la sécurité des occupants. Le paiement d'une certaine somme au propriétaire rendait toute éviction illégale. Aujourd'hui, le gouvernement cherche à amender cette loi afin de renforcer les droits des occupants et notamment de les protéger de manière plus efficace des expulsions. L'amendement rend l'expulsion plus difficile car il impose entre autres d'obtenir une décision administrative préalable. Par ailleurs, il empêche de vendre la terre sans l'accord des locataires.

Ce projet, présenté au Parlement en février 2008, a suscité une forte émotion au Buganda et une importante mobilisation de la part des royalistes ganda. Certains Baganda y voient une manœuvre pour déposséder le royaume de ses terres puisque la loi donne du pouvoir à des occupants (parfois illégaux) non ganda au détriment de propriétaires ganda. Ils accusent le royaume de favoriser l'occupation illégale de terres par des groupes non-ganda (notamment les Balaalo). Le Kabaka possède personnellement beaucoup de terres. L'amendement est donc également interprété comme une tentative d'humilier le Kabaka. Enfin, un certain nombre de Baganda, notamment des militants et des journalistes, accusent le gouvernement de chercher à 
démanteler le royaume en voulant restituer des terres données par les Britanniques à des Baganda au début du siècle à d'autres groupes ethniques.

Ces débats ont parfois favorisé l'expression de convictions autochtonistes ${ }^{21}$. La vente de terres appartenant à des Baganda à de nouveaux propriétaires non-ganda est interprétée comme une dépossession. Certains Baganda considèrent les nouveaux acheteurs comme des "étrangers » et considèrent qu'ils ne doivent pas posséder de terre sur un territoire correspondant au royaume du Buganda. Les ethnies de l'Ouest, dont sont issus la majorité des membres du gouvernement (et le président), sont particulièrement visées. Les "Westerners", parfois appelés "Rwandais " ou «longs nez » sont accusés de vouloir affaiblir le Kabaka en le dépossédant. Les vieux débats sur les supposées origines rwandaises de Museveni sont remis au goût du jour par des politiciens ganda qui professent des soupçons sur sa loyauté par rapport à l'Ouganda. Beti Kamya, pasionaria de la cause ganda, a ainsi publié un article intitulé «Où se situe le cœur de Museveni? $\aleph^{22}$. Le projet de loi sur la double nationalité, interdite jusqu'alors, a également ravivé des sentiments anti-rwandais présents au Buganda depuis la colonisation ${ }^{23}$.

\section{La mobilisation et les arrestations de juillet 2008}

Le 31 décembre 2007, le Kabaka Ronald Mutebi recompose son gouvernement ${ }^{24}$. Arrive à la tête du royaume une jeune génération de politiciens du royaume, avec parmi eux de jeunes avocats. L'équipe est fin prête pour la mobilisation mais aussi la négociation avec le gouvernement central.

La mobilisation autour de la sauvegarde de la forêt de Mabira, en 2006-2007, a déjà permis de créer un appel d'air sur ces thématiques. Mengo ${ }^{25} \mathrm{~s}^{\prime}$ est exprimé contre le projet gouvernemental de vente d'une partie de la réserve forestière à une compagnie sucrière privée pour en faire une plantation de cannes. Le 18 décembre 2007, le Kabaka Mutebi avait refusé de rencontrer Museveni pour discuter des questions de réformes foncières. S'en est suivi un échange de lettres entre le président et le Kabaka, missives qui ont été publiées dans la presse. Le 22 décembre 2007, Museveni envoie une lettre au Kabaka, dans laquelle il exprime son inquiétude face aux « intrigues, à la mauvaise foi et aux tendances séditieuses » observables à Mengo. Le 29 arrive la réponse de Mutebi, qui expose ses griefs contre la loi sur les terres et se distancie des partis politiques ${ }^{26}$.

On assiste au lancement de campagnes concurrentes d'information et de sensibilisation sur le thème des questions foncières. Le royaume et le gouvernement organisent des réunions et des ateliers de formation et d'explication sur le projet de loi, entament des tournées en zones rurales, investissent les médias. Les ebimeeza, ces débats radiophoniques en plein air de Kampala, sont investis par les partisans des deux camps.

Le 2 novembre 2008, le Kabaka a mis en place un Comité d'éducation civile (CCEC) chargé d'organiser la mobilisation contre la réforme foncière. À sa tête est placé Betty Nambooze. Porte-parole du Democratic Party ${ }^{27}$, la jeune femme a auparavant écrit dans des journaux comme The Shariat et Bukedde. Tous les mercredis elle participe au très populaire talk show de CBS, la radio du royaume du Buganda (la plus écoutée à Kampala) "Take it or Leave it " (Kiriza Oba Gaana), présenté par Medi Nsereko. Son appartenance au DP fut très critiquée par les partisans du gouvernement. En effet, selon l'article 246 de la Constitution, un dirigeant culturel ou traditionnel ne doit pas participer à la politique partisane ${ }^{28}$. Il est également révélateur du rapprochement opéré entre 
l'institution royale et le parti, alors que les deux acteurs étaient de farouches adversaires au tournant de l'indépendance ${ }^{29}$. Des associations de jeunesse comme les Young Democrats, Nkoba Zambogo et Bazukulu ba Buganda ont également un rôle important dans la mobilisation contre la réforme de la loi sur les terres.

Du côté gouvernemental, le Media Centre, créé en 2005 dans la foulée de la campagne électorale en vue des élections de 2006, a joué un rôle important dans la diffusion d'informations sur les questions foncières. Il forme notamment des militants NRM à la prise de parole et les «briefe » sur la réforme afin qu'ils puissent sensibiliser l'opinion publique en participant à des débats et à des réunions d'information. Le 18 juillet 2008, à l'occasion du quinzième anniversaire du couronnement de Mutebi, eut lieu à Kampala la Conférence du Buganda, qui réunit des intellectuels et des militants pour une journée de réflexion autour de l'histoire du royaume et de sa place dans l'État ougandais. Ce même jour, le gouvernement ordonna l'arrestation de trois hauts fonctionnaires du royaume dont Betty Nambooze et Peter Mayiga, ministre de l'Information du Buganda. Alors que les gardes à vue ne doivent pas dépasser 48 heures, il était impossible d'obtenir pendant six jours des nouvelles de ces trois personnes. En réalité, les trois fonctionnaires ont été emmenés d'un poste de police à l'autre à travers le pays pendant une semaine, pour finalement être relâchés, avec cependant pour deux d'entre eux une accusation de sédition. Le gouvernement affirme que ces personnes préparaient une rébellion armée. La réaction de l'opinion publique, royaliste ou non, était prévisible. Ces violations grossières des procédures et des droits des personnes mises en cause ont été critiquées de manière virulente. Cette affaire a également réveillé des divisions au sein du NRM. Enfin, elle a fait des trois protagonistes, et en particulier de Betty Nambooze, de véritables porte-drapeaux de la cause. Après leur arrestation, on pouvait voir des badges et des posters à leur effigie en vente dans les rues de Kampala.

En avril 2009, une nouvelle polémique a éclaté, lorsque le gouvernement a offert 2 milliards de shillings au Royaume dans le cadre d'une campagne de lutte contre la pauvreté. Les débats ont fait rage parmi les notables ganda sur l'opportunité d'accepter ou non cet argent. Certains ont interprété ce geste comme une tentative de corruption et de modération des revendications du royaume sur les questions foncières et le débat autour du fédéralisme. 350 millions ont tout de suite été versés et acceptés ${ }^{30}$.

Le dernier épisode en date est la reprise du projet de création d'une Autorité de la ville de Kampala, qui viendrait remplacer l'actuel Conseil de la ville de Kampala (KCC), un projet déjà dans les cartons en 2005. Selon Mengo, cette réforme donnerait davantage de pouvoir au gouvernement central dans la gestion de la ville. Les formes de l'élection du maire seraient modifiées. La métropole sera agrandie pour comprendre des parties des districts voisins. Le gouvernement pourra aussi désigner un administrateur de la ville, qui sera placé sous l'autorité du président. Autrement dit, le pouvoir central prend davantage d'influence sur la gestion politique et économique d'une zone qui représente le cœur du territoire revendiqué par le Royaume. Il faut également prendre en compte que lors des élections, Kampala est majoritairement acquise à l'opposition (le maire est membre du DP). L'administration municipale a été entachée par des scandales de corruption, ce qui justifie une volonté de réforme de son gouvernement. La question autour de ces polémiques est simple et épineuse à la fois : qu'est-ce qui fait partie du Buganda ou pas? Comment le déterminer? 


\section{Enjeux politiques autour des autorités « traditionnelles »}

Dans un tel contexte de tension avec le royaume du Buganda, le gouvernement a tenté de s'assurer le soutien d'autres institutions néo-traditionnelles. La polémique a notamment éclaté autour du Buruuli, une zone située dans le district de Nakasongola (à une centaine de kilomètres au Nord de Kampala) ${ }^{31}$. Butamanya a été désigné chef des Baruli le 10 décembre 2004. Les Baruli sont reconnus par la Constitution de 1995 comme l'une des " communautés indigènes » de l'Ouganda. Certains d'entre eux cherchent à se distinguer du Buganda. Le Buruli a été donné au Buganda au début du siècle par les Britanniques en même temps que les autres "comtés perdus" mais il n'a pas été restitué en 1964.

Le 8 octobre 2008, le Kabaka devait se rendre à Nakasongola pour lancer un bulungi bwansi (travail communautaire) et célébrer l'anniversaire de l'indépendance. Des manifestations hostiles avaient été prévues et la visite a été fortement découragée par le gouvernement et la police. Une fois de plus, la question est : quelles sont les limites du Buganda? Mengo considère que cette zone fait partie du royaume et que le Kabaka devrait pouvoir s'y rendre sans en informer le chef des Baruli. Les militants ganda accusent Museveni de créer des chefferies et des royautés dans la zone afin d'affaiblir le Buganda. Selon l'accord de 1900, le Buganda est composé de 20 comtés, dont le Buruuli, qualifié aujourd'hui de "comté dissident ", certains habitants exprimant une volonté de sécession ${ }^{32}$.

Dans ce contexte, le règlement des problèmes fonciers du Bunyoro ${ }^{33}$ est un enjeu politique de premier ordre, d'autant plus que la région est devenue riche en pétrole depuis 2006. Là aussi, des débats aux relents autochtonistes font rage ${ }^{34}$. Un groupe de pression, le Mubende Banyoro Committee, demande que les migrations des Bakiga dans la zone soient régulées et que des limites soient mises à leur accès à des responsabilités politiques dans le district de Kibaale. Le comité demande aussi que le gouvernement récupère les titres de propriété de plusieurs centaines de propriétaires fonciers ganda absents et les redistribue aux occupants « indigènes » banyroro. Ces titres de propriété ont été donnés à des dignitaires ganda après la défaite de l'Omukama (roi) du Bunyoro, Kabalega, contre les Britanniques en 1899. Ces « comtés perdus » avaient été restitués au Bunyoro lors d'un référendum en 1964 mais appartiennent encore parfois à des propriétaires ganda. L'omukama Kabalega a par ailleurs été déclaré « héros national » par Museveni le 9 juin 2009. Le FDC a quant à lui également mobilisé dans la région en $2008^{35}$.

50 Le $1^{\text {er }}$ septembre 2008, le Kyabazinga (roi) du Busoga (Est du Buganda), Wako Muloki, est décédé. Sa mort a déclenché des débats autour du mode de désignation de son successeur. Doit-il être élu par un corps électoral de notables ou déclaré Kyabazinga à travers des rituels ? Doit-il y avoir rotation entre les différents clans d'appartenance du Kyabazinga? Des craintes d'interférences de la part du gouvernement central dans cette désignation ont également été exprimées ${ }^{36}$. Le 31 octobre 2008, 7 des 11 chefs royaux du Busoga ont choisi par consensus le prince Wambuzi Muloki comme nouveau Kyabazinga. Certains chefs s'opposent à son règne car ils disent que le trône devrait passer d'une chefferie à l'autre. Une plainte auprès de la Cour constitutionnelle avait été déposée le 27 octobre pour demander à ce que toutes les chefferies puissent être en compétition. La Cour avait émis un ordre suspendant l'élection, ce qui n'a pas été respectée ${ }^{37}$. 
51 Autre controverse autour du rôle politique des chefs coutumiers, en janvier 2009, le Forum des leaders africains traditionnels, organisé par Muammar Kadhafi et qui devait se tenir à Kampala a été interdit par les autorités. Le gouvernement a argué que la conférence risquait de prendre une tournure "politique», ce qui est anticonstitutionnel en Ouganda, et d'influencer les débats autour des modalités de l'intégration panafricaine, sur lesquelles Museveni et Kadhafi se sont affrontés cette année à l'Union africaine. Par ailleurs, la conférence incluait John Barigye, l'héritier de la couronne de l'Ankole, un autre royaume ougandais (Ouest du pays) que Museveni ne souhaite pas restaurer. Lors du sommet de l'UA à Addis Abeba en février 2009, une autre friction a eu lieu entre Museveni et Kadhafi au sujet de la place à accorder aux leaders traditionnels dans le processus d'intégration régionale. En mars 2008, lors de la visite de Kadhafi pour inaugurer la grande mosquée de Kampala, le président libyen avait fait une visite surprise au Kabaka, ce qui avait déplu aux officiels ougandais.

\section{La reprise de la guerre contre la LRA}

52 La fin de l'année 2008 a vu la remise en cause des espoirs de règlement pacifique du conflit entre le gouvernement ougandais et Joseph Kony suscités par les négociations de Juba, entamées en juillet 2006.

\section{Les négociations de Juba}

53 En juillet 2006, des négociations étaient entamées entre le gouvernement et la LRA à Juba, au Sud Soudan. À la tête de l'équipe de médiation se trouvait l'ancien président mozambicain, Joachim Chissano et Riek Machar, vice-président du Sud Soudan. Le 26 août était signé un accord mutuel de cessation des hostilités. De l'avis général, il s'agissait d'un des plus grands espoirs de voir le conflit entamé dès 1986 dans le Nord du pays se régler de manière pacifique. Plusieurs processus de négociations, en 1992 et en 2004, s'étaient déjà soldés par des échecs. Les dernières tentatives militaires d'en découdre n'avaient pas porté leurs fruits et s'étaient souvent terminées en désastres humanitaires, comme l'opération Iron Fist en 2002, menée en accord avec le gouvernement soudanais contre les bases de la LRA dans ce pays ${ }^{38}$. Cependant, la LRA ressortit des deux offensives Iron Fist (2002 et 2004) affaiblie. Le contexte était également favorable du fait du processus de paix soudanais (2005), de la mise en place de la Commission d'amnistie en 2000, de l'attention internationale croissante illustrée par l'implication de la Cour pénale internationale (CPI) et d'une certaine volonté politique d'en finir avec un conflit vieux de 20 ans (qui compromettait par ailleurs pour le gouvernement les espoirs de conquête électorale du Nord du pays). En décembre 2003, Museveni demanda à la CPI d'intervenir en Ouganda. En juillet 2004, la Cour entamait ses enquêtes. En octobre 2005, elle émettait 5 mandats d'arrêts internationaux, les premiers de son histoire, contre Joseph Kony et quatre de ses officiers.

54 La question du rôle de la CPI et des modalités de la gestion des questions de justice et de réconciliation ont été au centre de controverses entre les différents acteurs locaux (gouvernement, rebelles, ONG transnationales, groupes religieux). La plupart des militants de la paix engagés sur le terrain et dans les instances internationales poussaient à l'abandon des mandats et à la mise en place d'un système alternatif, 
«traditionnel » et non pénal de réconciliation, basé sur la cérémonie du mato oput ${ }^{39}$. Cette ligne était également celle des rebelles et plus particulièrement de Joseph Kony, qui conditionnait sa signature de l'accord de paix à un retrait des mandats d'arrêt. La polémique a considérablement ralenti le processus de négociation, notamment le chapitre 3 des discussions, relatif à la réconciliation et à la responsabilité (accountability).

Le non-respect, à plusieurs reprises, par les rebelles, des délais accordés pour rassembler leurs troupes à des points de cantonnement, la reprise ponctuelle de combats (notamment en octobre 2006), les fortes dissensions au sein de la LRA (qui ont culminé avec la mort de Vincent Otti en octobre 2007) ont également ralenti le processus de pai $\mathrm{x}^{40}$. Les négociations ont également été difficiles sur les questions de partage du pouvoir et sur les modalités d'intégration des rebelles dans l'armée nationale. Ces derniers demandaient au départ 5 postes de ministres et la conservation de leurs rangs militaires de la LRA au sein de l'armée nationale. Le gouvernement a finalement obtenu un accord sur des "politiques d'égalité des chances », s'engageant à instaurer un équilibre national au sein du gouvernement, de la fonction publique, de l'armée et des forces de sécuritét ${ }^{41}$.

Finalement, le 19 février 2008, un accord sur le fameux chapitre 3 a été trouvé. Il prévoit l'utilisation du mato oput et la création d'une division spéciale au sein de la Haute Cour ougandaise pour juger les crimes les plus graves. La création de cette Cour permettrait, dans l'esprit des signataires, de négocier avec la CPI le retrait de ses mandats, contrairement au seul mato oput. En effet, selon le statut de Rome, la CPI prend en charge des affaires que les juridictions nationales ne veulent ou ne peuvent pas prendre en charge elles-mêmes. Quant à savoir si la CPI est prête à retirer des mandats déjà émis, c'est une autre question. L'accord a été critiqué par les grandes ONG transnationales comme Amnesty International et Human Rights Watch, qui militent pour l'instauration d'une justice pénale au niveau international.

Le 22 février 2008, un cessez-le-feu permanent était signé. À ce moment-là, la paix était toute proche pour le Nord de l'Ouganda. Il ne manquait plus, pour que ce cessez-le-feu soit entériné, que la signature d'un Accord de paix final (FPA). La date de la signature, initialement fixée au 28 février, fut repoussée au 28 mars, puis au 10 avril 2008. Kony ne vint jamais signer, réfugié dans les forêts du parc national de la Garamba, en RDC et ouvrant de nouveaux fronts jusqu'en Centrafrique.

\section{L'opération « Lightning Thunder » (« Eclair et Tonnerre »)}

58 À la différence de l'opération de 1997, Lightning Thunder a été menée non seulement avec la bénédiction du gouvernement congolais mais en partenariat avec les FARDC. L'opération de 1997, officiellement menée pour écraser les rebelles des Allied Democratic Forces (ADF), avait été décidée de manière unilatérale, sans l'accord de Kinshasa ni des parlementaires ougandais. Si Kabila a donné son accord sur la présence de l'UPDF sur son territoire, les représentants des Ougandais n'ont eux, une fois de plus, pas eu leur mot à dire sur le déclenchement de l'opération. Il n'y a eu ni vote, ni de consultation du Parlement.

Après l'échec du 10 avril, un nouvel ultimatum a été lancé le 5 novembre 2008 lors d'un sommet réunissant les principaux protagonistes des négociations, y compris la délégation de la LRA. Kony devait venir signer l'Accord de paix final avant le 
29 novembre. Une fois de plus, les négociateurs essuient un refus du chef rebelle. Le 14 décembre 2008, l'exécutif ougandais lance une campagne de bombardements aériens contre les camps de la LRA en RDC, appuyés par l'armée congolaise et le SPLA. L'objectif est de forcer Kony à signer l'accord.et de réduire à néant la capacité guerrière de la LRA en détruisant les camps situés dans le parc national de la Garamba. L'offensive ne durer que quelques jours. Elle a finalement duré trois mois. Plusieurs chefs rebelles ont été capturés ou tués pendant les opérations. En mars, le porte-parole de l'armée parlait de 350 morts parmi les rebelles, information qui est difficile à vérifier ${ }^{42}$.

Si la coopération militaire entre la RDC et l'Ouganda dans le cadre de cette opération témoigne d'un indéniable réchauffement des relations entre les deux pays, la prolongation de l'autorisation de combattre sur le territoire congolais ne s'est pas faite sans tensions. Au départ, le mandat ne devait durer qu'un mois. La dead line a été repoussée à deux reprises, en janvier et en février sous pression de Kampala ${ }^{43}$. Finalement, le retrait a été opéré à partir du 15 mars après une rencontre entre Kabila et Museveni à Kasese le 28 février. L'opinion publique congolaise devenait de plus en plus hostile à la présence de troupes étrangères sur son territoire.

61 L'opération a été marquée par les «massacres de Noël », commis par la LRA dans les villages de Doruma, Faradje, Gurba et Dungu. Selon Human Rights Watch, 620 civils ont été tués et 160 enfants enlevés entre le 24 décembre 2008 et le 13 janvier $2009^{44}$. Différentes ONG ont rapporté des attaques sur des églises et des rassemblements organisés dans le cadre des fêtes de fin d'année. Elles ont également signalé la présence de près de 100000 déplacés congolais dans la région de Dungu ${ }^{45}$. En Ouganda et à l'étranger, "Lightning Thunder» a été moins critiquée que les autres opérations militaires ougandaises, bien qu'elle remette en cause les espoirs de négociations futures et de règlement pacifique du conflit avec Kony. Non seulement elle a été menée avec l'accord de la RDC, mais elle n'a pas eu de conséquences sur le terrain en Ouganda, contrairement à "Iron Fist » (« Poing d'acier »), au Soudan qui avait poussé les rebelles à revenir en Ouganda et à s'attaquer aux populations civiles. Pour certaines ONG, tout espoir de négociation avec Kony était déjà abandonné du fait de l'attitude du chef rebelle. Une ONG américaine a appelé à reprendre l'offensive après le retrait du 15 mars $^{46}$. À Kampala, les journalistes ont davantage critiqué les erreurs tactiques de l'état-major, une certaine inefficacité finale de l'opération (Kony court toujours) et le manque de transparence quant au nombre de victimes des deux côtés plutôt que le bien-fondé de l'opération militaire.

\section{La situation des réfugiés et des déplacés}

62 Selon le HCR, dans un rapport de 2007, les déplacés du Nord de l'Ouganda étaient 1,6 million, dispersés dans des dizaines de camps ${ }^{47}$. Selon l'OCHA, le Bureau de la coordination des affaires humanitaires de l'ONU, en février 2009, il ne restait que $30 \%$ des déplacés dans les camps ${ }^{48}$. Les opérations de retour ont débuté au pays lango dès la signature de l'accord de cessation des hostilités, en août 2006. Selon le HCR, entre novembre 2006 et juin 2007, 500000 personnes sont rentrées dans leur village dans le Nord de l'Ouganda ${ }^{49}$.

63 Ces retours, encouragés par les bailleurs, le HCR, les ONG et le gouvernement, ont donc été réalisés de manière rapide. Ils ne sont pas sans poser de sérieux problèmes, notamment au niveau de la réattribution des terres. Des débats ont également eu lieu 
sur leur caractère obligatoire ou non, le gouvernement cherchant à normaliser la situation le plus rapidement possible alors que certains habitants se montraient réticents à rentrer, craignant une reprise de la guerre. La question du retour pose inévitablement des questions de recomposition de la politique et de l'économie locale. Les besoins des populations locales restent importants y compris en dehors des camps. Certaines opérations de rapatriement ont été ralenties par la dernière opération en $\mathrm{RDC}$, car les déplacés avaient peur d'une reprise de la guerre sur le territoire ougandais. Par ailleurs, depuis mai 2006, des milliers de réfugiés soudanais sont rentrés dans leur pays $^{50}$. En 2007, ils étaient plus de 200000 sur le territoire ougandais ${ }^{51}$.

Cependant, ces derniers mois, de nouveaux réfugiés ont passé la frontière ougandaise pour fuir le conflit entre le gouvernement congolais et le général rebelle Laurent Nkunda. Le HCR, dans un communiqué de janvier 2009, évalue à 50000 réfugiés le nombre de réfugiés congolais en Ouganda, majoritairement installés dans les camps de Matanda et Nakivale. Ces déplacements de population se sont intensifiés lors des dernières opérations menées par l'armée rwandaise contre Nkunda. L'Ouganda a accueilli 27000 réfugiés supplémentaires entre août et novembre $2008^{52}$.

\section{L'opposition et la répression}

La mobilisation pour la préservation de la forêt de Mabira, en 2007, avait permis aux différentes forces de l'opposition de se rassembler et de mesurer l'ampleur de leur potentiel contestataire dans l'unité. En effet, différents partis, mais aussi des ONG locales et transnationales, des groupes professionnels (associations de commerçants notamment), certains médias, des groupes religieux et des représentants d'autorités néo-traditionnelles, s'étaient regroupés en une "Save Mabira Crusade», afin de contester un plan gouvernemental de cession à un groupe sucrier privé d'une partie de cette forêt protégée. Cette mobilisation trans-sectorielle avait porté ses fruits, puisqu'en octobre 2007, le gouvernement a officiellement renoncé au projet ${ }^{53}$. Cette unité de l'opposition au gouvernement aura été de courte durée. Du fait de ces difficultés internes et de leur criminalisation par le gouvernement, les partis d'opposition ont encore du mal à trouver leurs marques et surtout à s'unir. La presse continue à faire preuve de vitalité. Elle joue un rôle central dans la dénonciation des scandales politico-financiers. Cela, malgré les opérations de reprise en main de la presse publique par le gouvernement et le lancement d'une importante machine de communication gouvernementale depuis la campagne électorale de 2006. La scène judiciaire reste l'un des espaces centraux d'affrontement entre le gouvernement et ses contradicteurs, avec plus ou moins de succès pour ces derniers.

\section{Les difficultés de l'opposition partisane}

Le principal parti d'opposition, le Forum for Democratic Change (FDC) n'est pas exempt d'affaires sulfureuses ${ }^{54}$. Le 20 juin 2008, Sulaiman Kigundu, «chairman » national du FDC, décède en Afrique du Sud. Sa mort déclenche des conflits autour des modalités de sa succession. Les 7 mois qui ont séparé la mort de Kigundu et la conférence des délégués du parti de février 2009 ont été marqués par des controverses sur les critères politiques, religieux et ethniques à appliquer dans le choix afin de respecter un principe de représentativité nationale du parti. 
des tentatives peu fructueuses des différents partis d'opposition de former un front commun, le Protocole ougandais sur la coopération inter-partis. Pendant l'année, un certain nombre d'élections partielles ont été remportées par le NRM parce que l'opposition était divisée, ce qui pourrait bien servir d'élément déclencheur à des tentatives plus fructueuses de mise en place de coalitions, voir d'un accord sur un candidat commun pour la présidentielle de 2011. Cependant, le Democratic Party, la formation d'opposition la plus importante après le FDC, particulièrement influente à Kampala, dans l'Ouest du Buganda et dans le Nord a finalement refusé de signer l'accord. Le PPP, parti du vétéran politique Jaberi Bibandi Ssali, a également refusé cette alliance, par peur de disparaitre au profit des plus grosses formations partisanes comme le FDC. Finalement la coalition du 5 août rassemble 4 partis: le FDC, l'Uganda Peoples Congress (UPC, ancien parti de Milton Obote), le Conservative Party (CP) et le Justice Forum ${ }^{56}$.

71 En mai 2008, la Cour constitutionnelle a rendu son verdict sur la question de la légalité des manifestations de rue et statué qu'il n'y avait pas besoin de l'autorisation de la police pour manifester. La Cour a annulé une section de la loi sur la police qui permettait à l'Inspecteur général de la police d'interdire une manifestation s'il estimait que celle-ci pouvait causer du désordre ${ }^{57}$. Cette décision a été considérée par l'opposition comme une victoire importante. soupçonnés de faire partie d'un nouveau groupe rebelle basé dans le Nord du pays, le Popular Patriotic Front (PPF). Les députés d'opposition ont réagi en faisant part de leurs craintes que le gouvernement crée un nouveau groupe rebelle dans la visée des élections de 2011, comme cela est soupçonné pour celles de 2006 avec l'Armée de rédemption du peuple (PRA) ${ }^{58}$. Le 14 décembre 2005, le leader du FDC Kiiza Besigye 
avait été arrêté en pleine campagne électorale sous le prétexte de son appartenance au PRA.

\section{La vitalité des médias malgré la dégradation de leurs conditions de travail}

Le paysage médiatique ougandais a été marqué ces derniers mois par le lancement du magazine hebdomadaire The Independent en décembre 2007. Le journal a été créé par Andrew Mwenda, ancien chef du service politique du Monitor et célèbre présentateur du talk show «The Andrew Mwenda Live » sur KFM. Mwenda avait quitté The Monitor de manière fracassante en août 2007. Sa lettre de démission, publiée dans le Weekly Topic, critiquait une main mise de l'actionnaire majoritaire, l'Aga Khan, sur le journal et la volonté de ce dernier d'épargner Museveni du fait de l'importance de ses investissements dans le pays ${ }^{59}$.

L'autre fait marquant dans l'histoire médiatique ougandaise de ces trois dernières années est la tentative de reprise en main du journal public The New Vision, qui parvenait jusqu'ici à maintenir une ligne éditoriale autonome. La campagne électorale de 2005-2006, qui a vu le départ de William Pike, à la tête du journal depuis 1986, a marqué un tournant à ce sens. En octobre 2008, la nouvelle rédactrice en chef Els de Temmermans a elle aussi quitté la rédaction, arguant du manque de liberté éditoriale ${ }^{60}$. Le Media Centre, placé directement sous l'autorité de la présidence et créé en 2005-2006 a servi, surtout à ses débuts, de nouvel organe d'encadrement du travail des journalistes. L'affaire de Blake Lambert, journaliste canadien proche du Monitor, s'est envenimée en mars 2006 lorsqu'il a été refoulé aux frontières ${ }^{61}$. Le gouvernement a également créé deux nouvelles radios, Vision Voice et Embuutikizi, qui partage du personnel avec le Media Centre. La nouvellement installée Nation TV, détenue par l'Aga Khan, a connu des difficultés lors de son installation en 200762. Enfin, la radio du royaume du Buganda, $C B S$ a été au centre de polémiques après que Museveni l'ait violemment critiquée en août 2008, qualifiant ses émissions de "stupides ${ }^{63}$.

Plusieurs journalistes de The Independent ont été brièvement arrêtés en avril 2008. En mai, les locaux du journal et la maison d'Andrew Mwenda ont été visités par la police. Ces arrestations sont survenues après la parution d'une série d'articles sur les «safe houses » en avril 2008. Les "safe houses " sont des centres de détention non déclarés où les services de sécurité procèdent à des interrogatoires, notamment de personnes accusées de terrorisme et de rébellion. Trois journalistes ont été accusés de sédition : Mwenda, John Njoroge et Charles Odoobo Bichachi. Mwenda et Njoroge ont été récompensés cette année par des prix internationaux de journalisme ${ }^{64}$.

En juin 2008, les locaux du tabloïd The Red Pepper ont été incendiés. Les auteurs de l'incendie ne sont pas connus. Cependant, le journal a été attaqué par des hommes véhiculés, masqués et équipés d'armes à feu. Le raid n'a duré que 15 minutes. Les attaquants s'en sont particulièrement pris à l'imprimerie du journal. Tous ces éléments ont conduit le management du Red Pepper à penser à l'implication d'une agence de sécurité ${ }^{65}$.

77 En juin 2009, la Cour constitutionnelle a décidé de maintenir la loi pénale sur la diffamation (section 179 de la loi sur le code pénal) et a affirmé qu'elle n'allait nullement à l'encontre de la Constitution ${ }^{66}$. La procédure fait partie d'une mobilisation de long terme de journalistes et d'avocats visant à dépénaliser les délits de presse, et 
notamment la diffamation (qui peut également faire l'objet d'une procédure civile prévue dans la loi ougandaise). La plainte auprès de la Cour avait été déposée par des journalistes du quotidien privé The Monitor. Ces derniers avaient été accusés de diffamation suite à la publication d'une enquête en août 2007 concernant le salaire de l'IGG $^{67}$. Une procédure similaire avait conduit, en 2004, à l'annulation de la loi sur la publication de fausses nouvelles, également présente dans la loi sur le code pénal. De la même manière, deux journalistes du Monitor avaient porté cette section devant la Cour constitutionnelle. Une plainte du même acabit portant sur le délit de sédition est encore en jugement.

\section{La question homosexuelle en Ouganda}

Depuis une dizaine d'années, la question de l'homosexualité a acquis une visibilité importante dans l'espace public ougandais du fait de sa dénonciation par des membres $\mathrm{du}$ gouvernement et des acteurs religieux, mais aussi en raison de l'institutionnalisation croissante des modes de protestation contre les discriminations et les violences dont les homosexuels font l'objet dans ce pays. Les militants dénoncent les mauvais traitements dont ils sont victimes, qui vont de la discrimination à l'embauche aux violences physiques, notamment de la part des autorités, en passant par le harcèlement, les humiliations, le rejet familial et social, le rejet par les institutions médicales qui a pour conséquence de bloquer l'accès aux soins et notamment aux antirétroviraux ${ }^{68}$, voire le viol ${ }^{69}$.

\section{La législation et l'action du gouvernement}

Depuis la fin des années 1990, le président Museveni ne cache pas ses sentiments antigays, encourageant les services de police à arrêter les homosexuels ${ }^{70}$. En Ouganda, l'homosexualité est considérée comme un crime et passible d'une peine d'emprisonnement à vie, selon la section 145 de la loi du code pénal. Cette loi affirme que toute personne ayant "une connaissance charnelle de toute personne contre l'ordre de la nature; une connaissance charnelle d'un animal ou; permet à une personne de sexe masculin d'avoir une connaissance charnelle de lui ou d'elle contre l'ordre de la nature commet un crime passible d'emprisonnement à vie. »Cette loi pose un certain nombre de problèmes en termes d'interprétation et d'économie de la preuve. L'acte sexuel est illégal mais pas le fait d'être homosexuel et de se revendiquer comme tel, rendant la répression des médias et des militants malaisée.

La section 148 de la même loi parle d'acte "d'indécence flagrante " sans définir ce à quoi cette notion fait référence. Cette section a été retirée du code pénal britannique ${ }^{71}$, qui a servi de modèle pour la rédaction de cette loi. Le terme homosexualité n'apparaît pas en tant que tel dans le code pénal ougandais ${ }^{72}$. À notre connaissance et selon les militants que nous avons rencontrés, personne n'a été condamné au nom de ces provisions du code pénal depuis les débuts du régime de Museveni ${ }^{73}$. D'où la volonté du ministre de l'Éthique et de l'Intégrité, Nsaba Buturo, d'adapter la législation.

En 1998, «Brenda », un transsexuel, a été condamnée pour avoir " causé du désordre ». Lors de son procès, le juge a déclaré qu' « il n'y a pas de loi en Ouganda qui interdit de s'habiller selon les codes de l'autre sexe ». Brenda n'a donc pas été condamnée sous des charges criminelles mais civiles ${ }^{74}$. En 2004, la radio privée Simba a été condamnée par le 
Conseil de la radiodiffusion à payer une amende de 1,8 million de shillings (720 euros) pour avoir accueilli des homosexuels dans un de leurs talk shows. Selon le secrétaire du Conseil, la radio avait enfreint la Loi sur les médias électroniques, qui interdit la diffusion de propos jugés contraires à la moralité publique ${ }^{75}$. En août 2007, le célèbre présentateur de radio Gaetano Kaggwa et son réalisateur ont été suspendus d'antenne pendant une semaine par le Conseil de la radiodiffusion. Les deux hommes avaient fait passer à l'antenne une jeune femme se présentant comme lesbienne, Victor Juliet Mukasa. Le Conseil accusa la radio d'être «vulgaire » ${ }^{76}$. Le 5 juin 2008 eut lieu une Conférence sur le sida à Kampala, organisée par UNAIDS et d'autres bailleurs internationaux. Les associations de défense des homosexuels n'avaient pas été invitées. De manière générale, les programmes nationaux de lutte contre le sida ne les intègrent pas dans les populations cibles. La plateforme des organisations homosexuelles en Ouganda, Sexual Minorities Uganda (SMUG) a du coup décidé de pénétrer dans les locaux qui abritaient la conférence afin de tenir une manifestation et de demander à être intégrés dans les discussions. Ils ont été arrêtés, mais là encore, le flou autour des dispositions légales sur l'homosexualité a pu être exploité. Les militants étaient accusés d'entrée illégale (criminal trespass) dans le lieu où se tenait la conférence. Les organisateurs ont alors rédigé une invitation pour SMUG, ce qui permettait d'aller à l'encontre des accusations de contrariété (annoyance) à l'encontre des organisateurs. Les militants ont été relâchés rapidement ${ }^{77}$.

Le dénouement de ces actions en justice illustre le fait qu'un certain flou existe dans la loi ougandaise en termes d'homosexualité. Cette imprécision permet de freiner les velléités répressives du gouvernement et des militants anti-gays sur le terrain du droit. Plus que de la loi, la répression dont souffrent les homosexuels en Ouganda est le résultat d'abus de pouvoir de la part des autorités et notamment du harcèlement des militants.

Nsaba Buturo cherche de ce fait à criminaliser le militantisme pro-gay et pas seulement les relations sexuelles avec une personne du même sexe. Il est souvent affirmé par les autorités que militer pour la reconnaissance des droits des gays ou se déclarer gay en public (où, selon le langage du gouvernement, "promouvoir l'homosexualité ", " enrôler ", " convertir » des jeunes) est illégal, ce qui est inexact. Le ministre cherche à faire évoluer la loi afin de pouvoir lancer des procédures contre les militants, gays ou non, qui organisent des manifestations et des conférences de presse sur le thème de la défense des droits des homosexuels, ou encore contre les journalistes qui donnent la parole à ces personnes ${ }^{78}$. Déjà, en 2005 , un amendement constitutionnel a été passé stipulant que le mariage n'est légal que s'il est contracté entre un homme et une femme.

Les discours hostiles à l'homosexualité se basent sur plusieurs types d'arguments. D'abord on trouve les discours religieux, basés sur des interprétations de la Bible et du Coran. Ces discours sont particulièrement portés par des pasteurs born again et le ministre de l'Éthique, lui-même pentecôtiste, mais également par des membres de l'Église anglicane d'Ouganda. Le débat est aussi lié à l'épidémie du sida, qui, selon UNAIDS, touche aujourd'hui entre 6 et $7 \%$ de la population ${ }^{79}$. Enfin, sont constamment invoqués des cas de viol dans les internats.

85 Avec celui de la religion, l'argument anti-gay le plus important est celui avançant une importation néo-coloniale de l'homosexualité. Nsaba Buturo parle de «mentalité de la traite » ou de « mentalité coloniale » pour désigner les militants des droits de l'homme 
qui défendent les homosexuels. Il affirme que l'homosexualité n'existait pas en Afrique avant la colonisation et que les associations locales et transnationales de défense des droits de l'homme importent des valeurs occidentales. Le soutien des organisations locales par des ONG internationales est assimilé à une satellisation. La question est présentée comme un débat sur les abus des conditionnalités de l'aide au développement, l'Ouganda s'attirant les foudres des bailleurs sur ces questions. L'idée que l'homosexualité est promue par les Européens en Afrique pour limiter les naissances sur le continent est très présente dans les discours des dirigeants et de certains citoyens. Ainsi, un auditeur d'une émission de radio consacrée à ce sujet affirmait que «la Banque mondiale finance l'homosexualité pour réduire le taux de croissance de la population » en Afrique. Des propos qui reprennent ceux de Museveni, qui encourage une politique de natalité ${ }^{80}$. D'où l'importance pour certains militants et chercheurs de démontrer un caractère "autochtone", "africain ", ou en tout cas précolonial de ces pratiques $^{81}$. Les militants essaient de défendre l'idée que l'homosexualité est africaine et que ce sont les lois qui la condamnent qui sont issues de codes pénaux coloniaux.

\section{Les mobilisations pro-gay}

Avant la mobilisation des homosexuels eux-mêmes, certains journalistes se sont engagés à couvrir et à défendre la cause homosexuelle en Ouganda, malgré un climat général d'hostilité dans la presse ${ }^{82}$. On peut citer notamment Andrew Mwenda, mais aussi certains des rédacteurs d'éditoriaux du New Vision.

D'autres intellectuels se sont engagés individuellement de manière intense. Sylvia Tamale est militante des droits humains, et notamment des droits des femmes depuis le début des années 1990. Elle est professeure à l'Université de Makerere et a récemment été élue doyenne du département de droit. Elle ne fait pas partie de ces associations, mais selon ses propres termes, elle les soutient et les conseille. Sa position de professeur lui a permis de donner une plus grande crédibilité à la cause même si elle doit supporter les coûts de son engagement, étant la cible de fréquentes attaques verbales dans les médias ${ }^{83}$.

Autre grande figure de la défense des homosexuels en Ouganda, Christopher Senyonjo, ancien évêque du diocèse du Buganda Ouest, a été excommunié dans les années 1990 de l'Église anglicane pour avoir défendu les homosexuels et contesté l'interprétation homophobe de la Bible. Il dirige l'organisation Integrity Uganda, qui organise des séances de conseil et offre un soutien moral aux victimes d'actes de haine homophobe et de discrimination.

Selon $\mathrm{M}^{\mathrm{me}}$ Tamale, avant le début des années 2000, «la lutte pour les minorités sexuelles venait par vagues, selon les incidents rapportés dans les médias. (...) C'était plus spontané. Les journaux publiaient par exemple qu'un couple s'était marié, ça déclenchait un débat et je venais les défendre. (...) [La] forme organisée de lutte politique, par les minorités sexuelles elles-mêmes, est relativement nouvelle ${ }^{84}$.» Auparavant, les organisations de LGBT se dédiaient au conseil et à la discussion plus qu'à la lutte pour la reconnaissance de droits. Les militants étaient tournés vers des activités de solidarité plutôt que vers la mobilisation. Ces premières organisations ont été créées dans la foulée des propos particulièrement hostiles de Museveni en 1999, et en réaction à la mise en place d'un plan national de lutte contre le sida dont les 
minorités sexuelles étaient exclues ${ }^{85}$. Dès 2000 , on observe un effort de coordination, avec la création de Sexual minorities Uganda (SMUG) qui rassemble plusieurs organisations. On observait alors également des formes de résistance individuelle, via la célébration de mariages, qui consistait en un échange de vœux et une fête, avec parfois, avant 2005 et l'amendement constitutionnel, l'intervention d'un pasteur étranger.

En 2004, après l'émotion suscitée par le suicide d'une jeune fille soupçonnée d'être homosexuelle en décembre 2003, plusieurs militants relancent la plateforme et décident d'affronter le gouvernement de manière plus directe, en cherchant ouvertement à changer son attitude envers les homosexuels et à se mobiliser contre la répression. Aujourd'hui, SMUG rassemble Freedom and Roam, l'association de Victor Juliet Mukasa, Spectrum Uganda, Integrity Uganda, une organisation religieuse créée par Senyonjo, et Icebreakers Uganda, une organisation de conseil et de discussion pour les jeunes ${ }^{86}$.

91 Victor Juliet Mukasa, une personne transgenre aujourd'hui chargée de recherche et de la politique pour l'Afrique de l'Est au sein du Centre et de la Corne pour la Commission internationale des droits des gays et des lesbiennes, est à la pointe de la mobilisation. Le 20 juillet 2005, son domicile fut attaqué par les autorités locales. Des papiers furent volés et une personne présente au moment des faits, Yvonne Oyoo, fut arrêtée et conduite au poste de police. Les policiers l'obligèrent à se déshabiller pour " prouver qu'elle est une femme ». Après cet épisode, les deux jeunes femmes décidèrent de déposer une plainte devant la Haute Cour au nom de l'article 50 de la Constitution sur l'application des droits fondamentaux et des libertés. La mobilisation investit la scène juridique. L'enjeu de cette plainte était la reconnaissance de l'égalité des droits des homosexuels.

De manière générale, l'année 2007 a été une année clé pour la mobilisation LGBT en Ouganda. Elle a d'abord gagné en transnationalisation. En janvier 2007, la participation d'une dizaine de militants de SMUG au Forum social mondial de Nairobi a permis un accroissement de la coordination avec d'autres organisations africaines et un accès décuplé au soutien d'organisations occidentales ${ }^{87}$. Pour les militants, il a aussi représenté une occasion d'apprentissage de techniques militantes, notamment concernant l'accès aux médias ${ }^{88}$. Selon un militant de SMUG, «le FSM a été l'un des points de départ du militantisme ougandais [pour les homosexuels]. Les Ougandais ont beaucoup appris à travers le $Q$ spot [atelier pro-gay du FSM]. Ils ont eu beaucoup d'idées et les ont ramenées ici. Ça a permis à beaucoup d'Ougandais de sortir du placard. Nous avons gagné en visibilité en nous attaquant à la presse et en disant: "venez nous rencontrer !" C'était après le FSM que nous sommes devenus si actifs. (...) Des campagnes comme la campagne médiatique que nous avons faite [en août 2007], nous recherchions de la visibilité et de la confrontation. (...) Via par exemple les rencontres organisées ici, la Journée mondiale contre le sida, la journée de la femme... ${ }^{89}$. Le FSM a également permis aux militants d'avoir accès à un réseau transnational en cas de répression et à de nouvelles sources de fonds. Depuis l'an dernier, SMUG bénéficie d'un bureau et d'une personne salariée en charge de l'administratif. Certains projets sont financés : une campagne sur les questions de sida, l'autre sur les questions policières.

Quelques mois plus tard, en mai 2007, au moment de l'audience de la plainte de Juliet Victor Mukasa et Yvonne Oyoo, des dizaines de personnes se sont mobilisées pour assister et manifester au procès de la Haute Cour. Le 16 août, à l'hôtel Speke de Kampala, eut lieu la première conférence de presse des personnes LGBT en Ouganda. À 
cette occasion, une dizaine de militants masqués ${ }^{90}$ ont parlé des problèmes de discrimination et de répression auxquels ils devaient faire face ainsi que du sida. Ils ont été rejoints par des militants kényans, un médecin et des membres de mouvements féministes et de lutte contre le sida locaux. Selon les organisateurs, l'idée était de "faire réaliser aux gens que nous n'avons pas été importés d'Occident ». La conférence de presse a été un succès, déclenchant de nombreux débats radiophoniques, des dizaines d'articles dans la presse, mais aussi une contre-mobilisation... Selon un militant du SMUG, la couverture médiatique de l'homosexualité s'est amplifiée et est devenue moins hostile qu'auparavant ${ }^{91}$.

En août 2007, une lettre de HRW au président Museveni lui reproche une politique de " condamnation d'État» de l'homosexualité en Ouganda9". La SMUG est maintenant soutenue au plan international par HRW, Amesty International, l'ILGA et l'International Gay and Lesbian Human Rights Commission. La plateforme est cependant peu soutenue par les autres organisations locales de défense des droits de l'homme, en dehors de quelques associations féministes. Ce manque de soutien local s'explique à la fois par l'homophobie ambiante et par la peur de la répression. Selon Sylvia Tamale l'Ouganda " a des lois draconiennes qui régulent les activités des ONG. Ces dernières ont peur de perdre leur statut. Alors certaines sont très prudentes. " Les militants locaux qui soutiennent les homosexuels le font davantage au niveau individuel, sans engager officiellement et ouvertement leur structure ${ }^{93}$.

En novembre 2007, SMUG organise une nouvelle manifestation lors du Commonwealth Head of Government Meeting (CHOGM), qui est réprimée par la police. Un an plus tard, le 22 décembre 2008, la Cour constitutionnelle a déclaré inconstitutionnels les actes des fonctionnaires de police et de l'administration commis à l'encontre de Juliet Mukasa et Yvonne Oyoo (perquisition illégale sans mandat, arrestation illégale, traitement humiliant, harcèlement sexuel) ${ }^{94}$. Selon la Cour, les mêmes droits constitutionnels s'appliquent aux personnes hétérosexuelles et homosexuelles, ce qui établit un important précédent juridique.

\section{Les mobilisations anti-gay}

Des acteurs de la société civile se mobilisent également contre l'homosexualité en Ouganda. Le tabloïd Red Pepper a publié à plusieurs reprises, en 2006 et en 2007, des listes d'homosexuels avec leur nom, le nom de leur partenaire présumé et l'endroit où ils habitent. Les acteurs les plus investis restent cependant les pasteurs born again. Certains dénoncent une « invasion systématique de l'homosexualité orchestrée par des puissances européennes et américaines ", ou comparent le «lobby homosexuel » à la mafia italienne ${ }^{95}$.

97 Le plus connu de ces militants anti-gay est le pasteur Martin Ssempa, ancien présentateur de talk show sur Monitor FM, en charge de l'Église de la Communauté de Makerere (l'Université de Kampala). Le 21 août 2007, il organise une manifestation anti homosexuels en réaction à la conférence de presse du SMUG du 16 août. Ssempa fonde la Coalition arc-en-ciel multiconfessionnelle contre l'homosexualité, qui appelle le gouvernement à être davantage sévère sur les questions homosexuelles. Les membres de la coalition reprennent l'idée d'une imposition de l'homosexualité par l'extérieur comme condition des prêts des bailleurs internationaux. Au-delà de la répression de ce qu'ils appellent la « promotion de l'homosexualité », la coalition cherche à remettre les 
homosexuels, considérés comme des pêcheurs et des malades, dans le «droit chemin » Ils organisent des ateliers de réhabilitation pour aider «ceux qui luttent contre leur attraction pour des personnes du même sexe " à faire rédemption. Pour les Églises pentecôtistes, la cause anti-homosexuelle est un moyen d'occuper la scène médiatique dans un contexte où la concurrence entre les Églises est forte ${ }^{96}$. Les pasteurs s'accusent d'ailleurs régulièrement entre eux d'être homosexuel.

L'Église anglicane prend également part à cette mobilisation. Lors de l'été 2008, on a assisté à la mobilisation de la branche ougandaise contre l'autorité de Canterbury. L'Église d'Ouganda conteste, avec d'autres églises africaines, la politique britannique concernant l'ordination des femmes et des homosexuels, menaçant de faire sécession.

\section{Conclusion}

Pour les différents acteurs politiques et sociaux ougandais, les derniers mois ont été marqués par la négociation d'alliances, en interne et en externe, dans la perspective des élections de 2011. Le NRM a vu le renforcement d'une opposition interne et la mise en place de courants qui s'appuient et s'affrontent sur fond de scandales financiers. Les partis d'opposition ont tenté de se rassembler en août 2008, sans succès. Au-delà de ces alliances en externe, on a vu que le FDC et le DP n'étaient pas exempts de conflits internes et qu'en leur sein, des coalitions et des courants sont également en cours de formation. Le royaume du Buganda a été bien souvent au centre de ces conflits et de ces renégociations d'alliances. La gestion des questions foncières, au Buganda comme ailleurs, apparaît comme étant l'un des enjeux cruciaux de l'échéance électorale.

Au-delà de ces affrontements politiques, les derniers mois ont été difficiles pour l'économie ougandaise. Tout d'abord du fait des répercussions de la crise postélectorale kényane, puis de celles de la crise financière mondiale, notamment au niveau monétaire. En Afrique subsaharienne, les effets de la crise mondiale peuvent se transmettre de plusieurs manières, à travers la baisse de la demande globale, les fluctuations de l'aide et la baisse des IDE via la hausse de l'aversion au risque ${ }^{97}$.

101 Dès janvier 2008, les conséquences des violences au Kenya se sont fait sentir, à travers d'importants problèmes de ravitaillement, en particulier en carburant. Les routes commerciales ont été coupées pendant plusieurs semaines. Les marchandises n'arrivant plus du port de Mombasa, les commerçants se sont retrouvés dans des situations difficiles. Les prix des produits de base ont augmenté. En effet, du fait de la hausse des prix de l'essence, les marchandises étaient difficiles à acheminer depuis les campagnes $^{98}$. Pendant la première semaine de janvier, dans les stations-service de Kampala, le prix d'un litre d'essence est passé de 2300 shillings (un peu moins d'un euro) à 6000 shillings (2,50 euros) $)^{99}$. Les queues s'allongeaient devant les stationsservice de Kampala. Au marché noir, le litre d'essence se vendait à 10000 shillings ${ }^{100}$.

102 À la fin de l'année 2008, les prix de l'essence à la pompe étaient toujours élevés, malgré la baisse globale du prix du baril ${ }^{101}$, du fait des dégâts sur le pipe-line Mombasa-Eldoret, les réductions sur les transports imposées par le Kenya et la hausse du dollar ${ }^{102}$. Le dollar a en effet atteint les 1950 shillings pour la première fois en trois ans, accroissant le prix des importations ${ }^{103}$. Entre 2008 et 2009, le taux d'inflation a énormément augmenté, passant à un nombre à deux chiffres, et atteignant les $14 \%$ en mars $2009^{104}$. Selon des observateurs locaux, en moins d'un an et demi, les prix de certains produits alimentaires comme la viande ou le matooke (bananes à la base de l'alimentation dans 
le Sud) ont quasiment doublé ${ }^{105}$. Le taux de croissance de 2009 projeté était de $8,1 \%$, il a été révisé à $6,2 \%^{106}$. Selon le comité parlementaire sur l'économie nationale, les apports des Ougandais de l'étranger ont baissé de $47 \%$ dans les 6 premiers mois de $2009^{107}$. Les activités boursières ont quant à elles chuté lors du troisième trimestre de $2008^{108}$.

Cependant, certains flux commerciaux paraissent s'être maintenus, voire avoir connu une certaine amélioration. Selon le Ministère des Finances, les recettes d'exportation ont été stables depuis l'année dernière. Lors de la première partie de l'année financière 2008-2009, toujours selon le ministère, ils atteignaient 1249,5 million de dollars, contre 1229,6 l'année précédente ${ }^{109}$. Les exportations de sucre, de café et de produits industriels ont augmenté entre 2008 et $2009^{110}{ }^{111}$. Le gouvernement a également annoncé l'ouverture de nouveaux marchés à l'exportation, notamment dans les pays émergents mais aussi dans l'Union européenne et aux États-Unis.

Les travaux sont toujours en cours sur le barrage de Bujagali, près des sources du Nil. Les explorations pétrolifères se poursuivent ${ }^{112}$, tout comme la construction d'infrastructures de stockage et de transport des hydrocarbures ${ }^{113}$. Le pays poursuit donc sa politique de tentative de réduction de la dépendance énergétique, même s’il reste importateur de pétrole. Le secteur des télécommunications est actif, notamment du fait de l'entrée sur le marché d'une nouvelle compagnie, Warid Telecom. La concurrence est ardue et les prix baissent, faisant augmenter régulièrement le nombre d'Ougandais ayant accès à la téléphonie mobile. Le nombre d'abonnés téléphoniques atteint les 7 millions en 2008 selon le ministère de l'Information ${ }^{114}$. L'économie reste cependant très dépendante des bailleurs de fonds internationaux. En janvier 2009, la Banque africaine de développement a annoncé le doublement de ses prêts entre 2008 et $2009^{115}$. C'est aussi sur ce type d'enjeux que se jouera le dénouement des élections de 2011 et que seront jugées les vingt-cinq années de régime NRM.

\section{NOTES}

1. F. Mugerwa, «Volunteer groups start fourth term campaign », The Monitor, 17/08/08.

2. E. Gyezaho, « NRM Mps declare Museveni 2011 candidate », The Monitor, 29/06/09.

3. A. Izama, « Otunnu attacks Museveni », The Monitor, 5/07/09.

4. T. Butagira, W.F. Okello, « No Votes, No Cabinet Posts-Museveni », The Monitor, 18/02/09.

5. S. Perrot, Le processus de reconstruction d'un ordre politique dans l'Ouganda de Y. Museveni (1986-2001): de la réversibilité du chaos ?, Thèse de doctorat de science politique, Université Bordeaux IV, 2003, en particulier pages 393-415.

6. Entretiens, Kampala, été 2008.

7. M. Nalugo, « SMS row threatens NRM party unity », The Monitor, 30/05/09.

8. G. Matsiko, S. Kibuuka, « How NRM historicals plan to oust Museveni », The Monitor, 28/06/08. 
9. S. Naturinda, «NRM Young Generation MPs face payback time ", The Monitor, 15/03/09.

10. Voir à ce sujet l'intéressant article de J. Buwembo, «Is There an Emerging Bahororo Mouvement?", The Monitor, 11/07/08.

11. Pour une analyse de l'affairisme militaire ougandais voir S. Perrot (1999), «Entrepreneurs de l'insécurité: la face cachée de l'armée ougandaise ", Politique africaine, 75, p. 60-71 ; A. B. Atuhaire, "How Kazini's $4^{\text {th }}$ Div became home of Ghosts ", The Monitor, 30/03/08.

12. S. I. Nganda, « Who Fought? Kashilingi Saved Museveni From Deadly UNLA Attack », The Weekly Observer, 17/06/09.

13. Voir les mémoires du Brigadier Pecos Kutesa (2006), Uganda's Revolution (1979-1986): How I saw It, Kampala, Fountain Publishers.

14. S. Sedigh, A. Ruzindana (1999), "The Fight against corruption in Uganda ", in R. Stapenhurst, S.J. Kpundeh Curbing Corruption. Toward a Model of Bringing National Integrity, World Bank Publications, p.179-206, voir p.184.

15. B. Among, F. Osike, "IGG orders Otafiire to explain Naguru ", The New Vision, 26/06/08.

16. Y. Mugerwa, M. Nalugo, "IGG SAGA : MPs apologize to Museveni ", The Monitor, 27/04/09.

17. La Constitution de l'Indépendance reconnaissait ce statut particulier du Buganda au sein de l'État ougandais. Pour une analyse du processus constitutionnel, voir P. Mutibwa (2008), The Buganda Factor in Uganda Politics, Kampala, Fountain Publishers.

18. Ibid., p. 245-250.

19. Voir le texte de l'accord sur http://www.buganda.com/buga1900.htm [Archive : https://web.archive.org/web/20190408095704/http://www.buganda.com/ buga1900.htm].

20. S.D. Doyle (2006). «From Kitara to the lost counties. Genealogy, Land and Legitimacy in the Kingdom of Bunyoro, Western Uganda ", Social Identities, 12 (4), p. 467-470.

21. J.-F. Bayart, P. Geschiere, F. Nyanja (2001), « Autochtonie, démocratie et citoyenneté en Afrique ", Critique internationale, 10, p. 177-194.

22. B. Kamya, « Where is Museveni's heart? », The Monitor 28/01/08.

23. Et sur lesquels Obote s'était appuyé pour chasser les Rwandais d'Ouganda en 1982. Sur les Rwandais en Ouganda, voir E.D. Mushemeza (2007), The Politics and Empowerment of Banyarwanda Refugees in Uganda 1959-2001, Kampala, Fountain Publishers.

24. Le Kabaka a autour de lui un cabinet composé de 31 ministres. Ces derniers ont des charges essentiellement symboliques, la Constitution déniant tout type de souveraineté politique au Royaume et les ressources pour faire fonctionner l'administration étant minces puisque le royaume ne peut lever d'impôts. Pour un point sur les modes détournés de formation d'un État au Buganda voir P. Englebert (2001), « Le Buganda. Un presque État dans l'État », Afrique contemporaine, $\mathrm{n}^{\circ}$ spécial, $3^{\mathrm{e}}$ trimestre, pp. 166-176. Voir la liste des ministres: https://web.archive.org/web/20090407034504/http:// www.buganda.or.ug/index.php?option=com_content\&view=article\&id=44\&Itemid=27

25. C'est ainsi que l'on désigne l'institution monarchique. 
26. Selon la Constitution, les royaumes ne peuvent se mêler de politique partisane, ce dont Museveni accuse le Buganda aujourd'hui.

27. Créé en 1954, le DP est le plus ancien parti d'Ouganda. Il est particulièrement implanté dans la région de Masaka, au Buganda, parmi les catholiques. Sans faire des scores aussi importants que le FDC, il bénéficie également d'un électorat relativement important dans le Nord du pays, majoritairement catholique. Voir H. Médard (2003). "La naissance et le déclin des partis politiques et religieux en Ouganda (1887-2002) ", Afrique et Histoire, 1, p.139-167.

28. Constitution of the Republic of Uganda, 1995, art. 246, section 3-e.

29. P. Mutibwa, The Buganda Factor in Uganda Politics, Kampala, Fountain Publishers, 2008.

30. R. Mwanje, «We won't reject government money », The Monitor, 25/06/09.

31. «President meets royal », New Vision, 9/12/08.

32. R. Mwanje, «Kabaka to launch Buganda Map », The Monitor, 25/10/08.

33. Le Buganda et le Bunyoro se sont affrontés (mais aussi alliés) à plusieurs reprises dans l'histoire de la zone. Pendant la colonisation, les Britanniques se sont appuyés sur les Baganda pour soumettre le royaume du Bunyoro. D'où en 1900 l'attribution de terres situées à ce moment là au Bunyoro à la couronne ou à des notables ganda. Pour l'histoire du Buganda au XIX siècle, voir $\mathrm{H}$. Médard (2007), Le royaume du Buganda au XIX ${ }^{e}$ siècle, Paris, Karthala. Voir en particulier les pages 155-182.

34. B. Kaija, A. Mugisa, "Museveni to sort out Bunyoro land rows", The New Vision, 10/06/09.

35. F. Mugerwa, «Bunyoro's search for her rightful place continues», The Monitor, 7/06/09.

36. S. Muyita, "Basoga oppose Museveni on Kyabazinga élections", The Monitor, 20/06/09.

37. « President meets royal », op. cit.

38. Pour un historique de la guerre dans le Nord de l'Ouganda voir S. Perrot (2004), "Vers une fin de conflit au Nord de l'Ouganda ? La Lord's Resistance Army (1987-2005), causes et enjeux d'une guerre prolongée ", Annuaire de l'Afrique orientale, p.73-139.

39. T. Allen (2006), Trial Justice. The International Criminal Court and the Lord's Resistance Army, London, Zed Books.

40. BBC, "Otti "executed by Uganda rebels" », 21/12/07, http://news.bbc.co.uk/2/hi/ africa/7156284.stm [Archive: https://web.archive.org/web/20190408100005/http:// news.bbc.co.uk/2/hi/africa/7156284.stm].

41. F. Nyakairu, «Counting the gains, losses of Juba peace talks», The Monitor, 9/03/08.

42. T. Butagira, « Highlights of Operation Lightning Thunder », The Monitor, 17/03/09.

43. B.H. Oluka, « How Kabila threw out UPDF troops », The Monitor, 17/03/09.

44. Rapport de HRW (2009) "The Christmas Massacres. LRA attacks on civilians in Northern Congo ", NY, HRW. Accessible à http://www.hrw.org/sites/default/files/ reports/drc0209web_0.pdf [Archive: https://web.archive.org/web/20130720185325/ http://www.hrw.org/sites/default/files/reports/drc0209web_0.pdf].

45. HRW, "ONU : Il faut déployer plus de casques bleus en RD Congo", 16/02/09; http://http:/www.hrw.org/en/news/2009/02/16/onu-il-faut-d-ployer-plus-de- 
casques-bleus-en-rd-congo [Archive : https://web.archive.org/web/20190408100346/ https://www.hrw.org/fr/news/2009/02/16/onu-il-faut-deployer-plus-de-casquesbleus-en-rd-congo].

46. J. Spiegel, N. Atama, Finishing the fight against the LRA, Strategy paper, Enough Project, 12/05/09, http://www.enoughproject.org/publications/finishing-fightagainst-lra-activist-brief [Archive: https://web.archive.org/web/20170205111551/ http://enoughproject.org/publications/finishing-fight-against-lra-activist-brief]

47. UNHCR, «Ouganda. Environnement opérationnel », Appel global 2007 de l'UNHCR, p.1 41-145.

48. http://appablog.wordpress.com/2009/03/18/ouganda-le-retour-des-personnesdeplacees-se-poursuit-dans-le-nord/ [Archive: https://web.archive.org/web/ 20190408100510/https://appablog.wordpress.com/2009/03/18/ouganda-le-retour-despersonnes-deplacees-se-poursuit-dans-le-nord/].

49. https://www.unhcr.org/fr/news/stories/2007/10/4acf01472a/ouganda-messagesradio-annoncent-deplaces-quils-peuvent-rentrer.html [Archive: https:// web.archive.org/web/20190408101144/https://www.unhcr.org/fr/news/stories/ 2007/10/4acf01472a/ouganda-messages-radio-annoncent-deplaces-quils-peuventrentrer.html].

50. https://www.unhcr.org/fr/news/stories/2009/1/4acf01db28/officiels-soudanaisappellent-refugies-ouganda-rentrer.html [ $\quad \mathrm{https} / /$ web.archive.org/web/ 20190408101053/https://www.unhcr.org/fr/news/stories/2009/1/4acf01db28/ officiels-soudanais-appellent-refugies-ouganda-rentrer.html].

51. UNHCR, « Ouganda. Environnement opérationnel », Appel global 2007 de l'UNHCR, p.141-145.

52. R. Russo, "Regain de violence en RDC, 13000 Congolais fuient en Ouganda ", UNHCR, Kampala, 27/11/08, https://www.unhcr.org/fr/news/stories/ 2008/11/4acf01c81c/regain-violence-rdc-13-000-congolais-fuient-ouganda.html [https://web.archive.org/web/20190408101000/https://www.unhcr.org/fr/news/ stories/2008/11/4acf01c81c/regain-violence-rdc-13-000-congolais-fuientouganda.html].

53. Pour une analyse détaillée et processuelle de cette mobilisation et des raisons de son succès, voir F. Brisset-Foucault (2007), «Des émeutes pour sauver la forêt de Mabira ", Mouvements, disponible en ligne: http://mouvements.info/des-emeutespour-sauver-la-foret-de-mabira/ [Archive: https://web.archive.org/web/ 20131110141058/http://www.mouvements.info/Des-emeutes-pour-sauver-laforet.html].

54. Sur les partis politiques en Ouganda, voir entre autres S. Makara (2009). "The Challenge of Building Strong Political Parties for Democratic Governance in Uganda : Does Multiparty Politics Have a Future ?", Les Cahiers de l'IFRA, 41, p. 43-80 ; G. Carbone (2003). « Political parties in "no party" democracy. Hegemony and opposition under "Movement democracy" in Uganda », Party Politics, 9 (4), p. 485-501.

55. R. Mwanje, «Wrangles delay DP delegates' conference », The Monitor, 23/06/09.

56. G. Bareebe, «Can the Opposition realise a single candidate strategy? », The Monitor, 24/08/08.

57. P. Amoru, « Uganda: Govt to Appeal Ruling On Public Démonstrations », The Monitor, 2/06/08. 
58. P. Amoru, S. Lawino, D. Okumu, « Rebel group: Who is telling truth?», The Monitor, 4/07/09 ; «Rebels talk: MPs demand answers », The Monitor, 26/06/09.

59. S. I. Nganda, « Mwenda quits Monitor, fires salvo at Aga Khan », The Weekly Observer, 23/08/07.

60. T. Butagira, « De Temmerman: Why I quit New Vision », The Monitor, 26/10/08.

61. Reporters sans frontières, "Comment mettre dehors un journaliste étranger", 3/04/06, https://rsf.org/fr/actualites/comment-mettre-dehors-un-journalisteetranger [Archive: https://web.archive.org/web/20190408101442/https://rsf.org/fr/ actualites/comment-mettre-dehors-un-journaliste-etranger].

62. CPJ, «In Uganda, government shuts down new TV station», 7/02/07, http:// cpj.org/2007/02/in-uganda-government-shuts-down-new-tv-station.php [Archive : https://web.archive.org/web/20171128212205/https://cpj.org/2007/02/in-ugandagovernment-shuts-down-new-tv-station.php].

63. S. Muyita, «Uganda: Museveni Attacks "Stupid" FM Radios", The Monitor, 24/07/08.

64. "The Independent journalists win two international awards", The Independent, $4 / 12 / 08$.

65. Committee to Protect Journalists, "Gunmen torch printing press of Ugandan tabloid », 1/07/08.

66. B. Tabaire, "Constitutional Court blows Chance to expand Free speech", The Monitor, 4/07/09.

67. Entretien avec James Nangwala, avocat, Kampala, août 2008.

68. A. Asiimwe, « Rights Body Pleads for Gay », The Monitor, 16/10/07, in S. Tamale, (ed.) (2007), Homosexuality. Perspectives from Uganda, Kampala, SMUG, p.144

69. Entretien avec un membre de Sexual minorities Uganda (SMUG), Kampala, septembre 2008; HRW, "Uganda: Press Homophobia Raises Fears of Crackdown», 7/09/06, http://hrw.org

70. J. Maseruka, «Arrest Homos, Says Museveni », The New Vision, 28/09/99, cité dans S. Tamale, op. cit., p.25

71. S. Tamale (2007), «Laws and Human Rights », in S. Tamale (ed.) (2007), op. cit., p.56.

72. S. Tamale (2007), «Laws and Human Rights", in S. Tamale, op. cit, p.55; Voir https://web.archive.org/web/20181006035031/https://ulii.org/ug/legislation/ consolidated-act/120 et https://web.archive.org/web/20170616000824/http:// www.ulii.org/ug/legislation/act/2007/8/

73. Entretien avec un militant du SMUG, Kampala, Septembre 2008.

74. E. Mulondo, «Brenda Gets Caution », The Monitor, 1/12/98, cité dans S. Tamale (ed.) (2007), op. cit., p.24-25.

75. R. Kagumire, «FM Station Penalised over Gays », The Monitor, 3/10/04, cité dans S. Tamale (ed.) (2007), op. cit., p.30.

76. A. Mugisa, «Gaetano Suspended over Homo Talk Show », The New Vision, 17/08/07, cité dans S. Tamale (ed.) (2007), op. cit., p.40-41.

77. Entretien avec Sylvia Tamale, Kampala, Septembre 2008.

78. Interview de Nsaba Buturo par R. Sserumaga, «Spectrum», Radio One, 2007, date exacte non indiquée. 
79.

http://data.unaids.org/pub/Report/2008/

uganda_2008_country_progress_report_en.pdf [Archive : https://web.archive.org/ web/20190408102318/http://data.unaids.org/pub/report/2008/

uganda_2008_country_progress_report_en.pdf].

80. J. Nandawula, « Museveni Opens War on Gay Men », The Monitor, 28/09/99, cité dans

S. Tamale (ed.) (2007), op. cit., p.27-28.

81. J. Siméant (2008), « Au Queer Spot. Notes de terrain », Vacarmes, 45.

82. Human Rights Watch, «Uganda Press Homophobia Raises Fears of Crackdown », 07/09/06.

83. Entretien avec S. Tamale, Kampala, septembre 2008.

84. Ibid.

85. Ibid.

86. Entretien avec un militant de SMUG, Kampala, septembre 2008.

87. M.-E. Pommerolle, J. Siméant (2008), « Voix africaines au Forum social mondial de Nairobi. Les chemins transnationaux des militantismes africains ", Cultures \& Conflits, 70, http://doi.org/10.4000/conflits.13423 ; S. Barris (2007), «Un autre monde est possible pour les personnes LGBT africaines ", ILGA - Mouvements, https:// web.archive.org/web/20160627055922/http://mouvements.info/un-autre-monde-estpossible-pour-les-personnes-lgbt-africaines/

88. Entretien avec un militant de SMUG, Kampala, Septembre 2008.

89. Entretien avec un militant de SMUG, Kampala, Septembre 2008.

90. Au-delà de la peur de la répression, les masques voulaient signifier le fait que n'importe qui pourrait être homosexuel, mais que les Ougandais ont peur de s'afficher en tant que tels.

91. Entretien, Kampala, septembre 2008.

92. HRW, « Letter to Ugandan President Regarding Homophobia and HIV », 21/08/07.

93. Entretien avec un militant de SMUG, Kampala, Septembre 2008.

94. FIDH, «Justice at last for Victor Juliet Mukasa! »23/12/08, accessible à https:// www.fidh.org/en/region/Africa/uganda/UGANDA-Justice-at-last-for-Victor [Archive : https://web.archive.org/web/20160920095802/https://www.fidh.org/en/region/ Africa/uganda/UGANDA-Justice-at-last-for-Victor]

95. G. Tegulle, « Homosexuality is Where the Money is », The Monitor, 15/09/07.

96. Voir à ce sujet les travaux d'A. Gussmann (2008), Complissità e Pentecotalismo a Kampala, Thèse de doctorat d'anthropologie, Université de Turin.

97. FMI, Perspectives économiques régionales. Afrique subsaharienne, Etudes économiques et financières, avril 2009, disponible sur http://www.imf.org/external/french/pubs/ft/ reo/2009/afr/sreo0409f.pdf [Archive : https://web.archive.org/web/20190408103220/ https://www.imf.org/ /media/Websites/IMF/imported-flagship-issues/external/ french/pubs/ft/reo/2009/afr/_sreo0409fpdf.ashx.

98. D. Nakawesi, «High Food, commodity prices take control of 2008 ", The Monitor, 31/12/08.

99. T. Butagira, A.Bagala, «Fuel Prices Double as Stock run out », The Monitor, 2/01/08 ; J. Nafula, A. Bagala, «Fuel Prices remain High, Petrol still scarce », The Monitor, 5/01/08. 
100. J. Nafula, A. Bagala, «Fuel Prices remain High, Petrol still scarce ", The Monitor, $5 / 01 / 08$.

101. C. Mwanguhya, « Editor's case note », The Monitor, 28/12/08.

102. P. Busharizi, « 2008. Year of Turbulence », The New Vision, 14/01/09.

103. P. Busharizi, « 2008. Year of Turbulence », The New Vision, 14/01/09.

104. http://www.lesafriques.com/ouganda/ouganda-baisse-du-taux-dinflation-a-13-4en- avril.html?Itemid=74? articleid=16011

105. D. Nakawesi, « High Food... », art. cit.

106. I. Musa Ladu, « Pain as URA reveals April Figures », The Monitor, 19/05/09.

107. Y. Mugerwa, « Kyeyo cash drops by Shs 500 billion », The Monitor, 19/06/09.

108. http://www.lesafriques.com/ouganda/la-bourse-d-ouganda-confronte-a-unebaisse- drastique.html? Itemid=74? articleid=11041

109. http://www.lesafriques.com/actualite/l-ouganda-ne-connait-pas-la- crise.html? Itemid=89?articleid $=15797$

110. http://www.lesafriques.com/ouganda/ouganda-les-exportations-ont-augmentede-37-en- mars.html?Itemid=74?articleid=16626

111. http://www.lesafriques.com/ouganda/ouganda-exportations-de-produitsagricoles-vers-les-usa- la-chine-l-inde-et.html?temid=74?articleid=14400

112. http://www.lesafriques.com/ouganda/ouganda-heritage-oil-et-tullow-oil-fontune-decouverte- de-petrole-de-classe-mond.html?Itemid=74?articleid=12633

113. http://www.lesafriques.com/ouganda/ouganda-un-gigantesque-reservoir-decarburant-sera- construit-pres-de-ka.html?Itemid=74?articleid=13768

114. W. Wafula, « Phone suscribers reach 7 million », The Monitor, 29/12/08.

115. http://www.lesafriques.com/ouganda/ouganda-la-bad-double-son-financementpour- 2009.html? Itemid=74? articleid=13813

\section{INDEX}

Index géographique : Uganda | Ouganda

\section{AUTEUR}

\section{FLORENCE BRISSET-FOUCAULT}

Florence Brisset-Foucault est doctorante en science politique et ATER (Attachée Temporaire d'Enseignement et de Recherche) à l'Université Paris 1 Panthéon-Sorbonne. Elle prépare une thèse sur la prise de parole politique, les registres de la critique et les formes de la citoyenneté dans l'Ouganda contemporain. Elle s'intéresse particulièrement à l'art oratoire en politique, à l'histoire et la sociologie des médias en Afrique de l'Est et a fait partie de plusieurs projets de recherche sur la politique électorale au Kenya et en Ouganda. 